\title{
V BIENAL DE ARQUITECTURA LATINOAMERICANA BAL2017 EN PAMPLONA
}

\author{
Rubén Labiano Novoa
}

\begin{abstract}
La celebración el pasado mes de abril de una nueva edición, la quinta, de la Bienal de Arquitectura Latinoamericana BAL2017 congregó en Pamplona a una selección de quince equipos de arquitectos jóvenes latinoamericanos, provenientes de siete países. Con la inclusión de los trabajos enviados por bienalistas de ediciones anteriores, fueron en total treinta y dos los equipos y ocho los países representados, convirtiendo a Pamplona por unos días en un escaparate del mejor presente de la arquitectura emergente latinoamericana.
\end{abstract}

Palabras clave: Bienal, Arquitectura, Latinoamericana, Pamplona, BAL Keywords: Biennial, Architecture, Latin American, Pamplona, BAL

La Bienal de Arquitectura Latinoamericana (BAL) celebró en 2009 su primera edición en un contexto mundial de crisis económica. Ocho años después, la celebración de la quinta edición refleja el logro de una iniciativa que ha sabido mantenerse, entre menguantes vaivenes presupuestarios, gracias a los patrocinios de la Universidad de Navarra, el Ministerio de Fomento, el Ayuntamiento de Pamplona, el Gobierno de Navarra y la Delegación en Navarra del Colegio de Arquitectos Vasco-Navarro.

Por encima de la supervivencia, siempre meritoria, esta edición ha supuesto la confirmación de la madurez alcanzada por un certamen que está logrando convertirse en el contrapunto al universo anglosajón establecido en su acta fundacional; un foro en el que estrechar lazos y acortar distancias, a pesar de celebrarse, o precisamente por celebrarse en un lugar absolutamente periférico con respecto al mundo latinoamericano. Condición periférica que en su día Luis FernándezGaliano calificó como extravagante ${ }^{1}$ y que sería una de sus notas características.

En esta edición la Bienal ha ampliado sus horizontes con el objetivo de obtener una mayor implicación entre la arquitectura y la sociedad a la que sirve. Manteniendo en la arquitectura su razón de ser, ha extendido su programa durante todo el mes de mayo a otros ámbitos de la cultura como el diseño, el cine, la fotografía, la gastronomía y la música latinoamericana alcanzando dimensiones propias de un auténtico intercambiador cultural. Conservando el esquema de exposición, presentaciones, mesas redondas y conferencias empleado en las pasadas ediciones y que constituye su núcleo duro, la BAL ha enriquecido su programa incorporando una sección de fotografía de arquitectura latinoamericana y acogiendo una itinerancia de la Bienal Iberoamericana de Diseño (BID_16). También la gastronomía ha encontrado su hueco en la Bienal organizando veladas gastronómicas con productos propios de cada uno de los países representados. Finalmente, las notas folclóricas con músicas y danzas autóctonas han aportado a la presente edición una visibilidad propia.

El programa arquitectónico, que es el que define el certamen, ha mantenido en Pamplona el esquema tradicional de funcionamiento de otros años y ha vuelto a contar con extensiones en Madrid y Barcelona. El país invitado este año fue Colombia, que protagonizó las conferencias y debates, centrados casi temáticamente en los conocidos logros sociales alcanzados en Medellín y Bogotá empleando estratégicamente la arquitectura como una eficaz herramienta de acción social.

El primer día de la BAL, tras las intervenciones oficiales de la Presidente del Gobierno de Navarra, Uxue Barkos, del Alcalde de Pamplona, Joseba Asirón, del Rector de la Universidad de Navarra, Alfonso Sánchez-Tabernero, del Embajador de Colombia en España, Alberto Furmanski Goldstein y del Presidente del Patronato de la Bienal Juan Miguel Otxotorena,

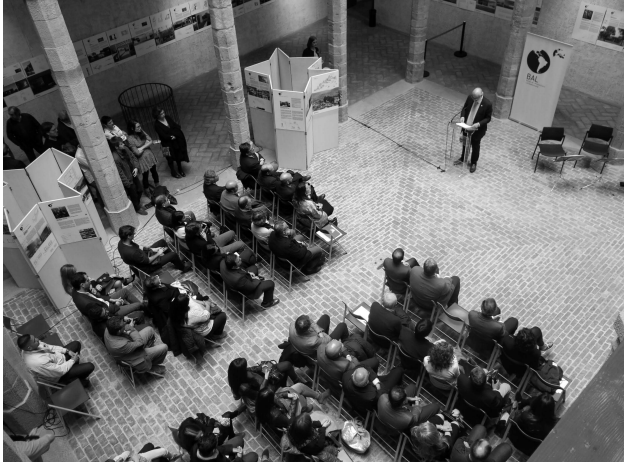

Fig. 1. Inauguración BAL2017. Embajador de Colombia.
1. Opinión expresada por Luis Fernández-Galiano en la mesa redonda celebrada en las Galerías de los Nuevos Ministerios de Madrid con ocasión de la IV edición de la BAL en 2015. 


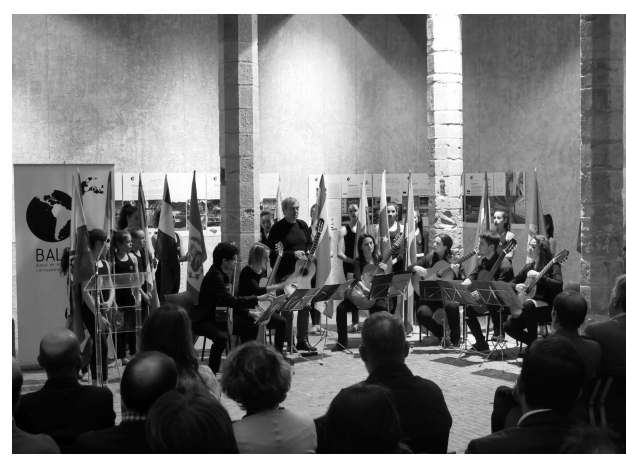

Fig. 2. Ceremonia de inauguración BAL2017. correspondió al arquitecto sevillano, y especialista en arquitectura iberoamericana José Ramón Moreno impartir la conferencia de apertura. Bajo el título "Un siglo de arquitectura en Colombia" Moreno disertó acerca del tránsito de la arquitectura colombiana a la modernidad que pensada en Europa se construyó en América Latina. Remontándose a sus orígenes academicistas impulsados desde finales del siglo XIX por la Escuela Nacional de Bellas Artes, Colombia asumió la modernidad gracias, según Moreno, a: "la triple aparición en 1905 de la Sección de Edificios Nacionales, la creación de la Universidad Nacional en Bogotá en 1935 y el primer número de la revista "Proa" en 1947"2. Estos serían los hechos cruciales para la entusiasta aventura que desterraría definitivamente la influencia academicista de las primeras épocas e ingresaría en un período decididamente orientado hacia el Movimiento Moderno. Ese período contó con numerosos protagonistas; notables profesionales que, formados de inicio en Europa, combinaron su ejercicio profesional con la docencia, consolidando una solidez conceptual y constructiva en el buen diseño que, sostiene Moreno, va a caracterizar la arquitectura colombiana hasta nuestros días. El más conocido de los arquitectos colombianos es, lo sabemos bien, Rogelio Salmona (19292007); pero, más allá de sus salmonistas descalzos, la eclosión de arquitectos colombianos que llega hasta hoy no se entendería sin aquella primera generación de arquitectos que les dio paso. Junto a sus maestros, la arquitectura colombiana se ha caracterizado por la presencia de gestores públicos inteligentes que han sabido dar nuevas oportunidades en Colombia a la arquitectura y a los arquitectos.

Tras el marco teórico establecido por Moreno la bienal prosiguió hablando de los gestores y maestros colombianos a los que antes aludíamos. En primer lugar, intervino uno de ellos, Carlos Mario Rodríguez Osorio, arquitecto y docente colombiano, que desde la posición que ocupó entre 2004 y 2011 como gerente de Diseño Urbano en la Empresa de Desarrollo Urbano de Medellín fue el responsable de la gestión de la mayoría de los proyectos urbanos del plan de desarrollo municipal de Medellín. Nos presentó la admirada, reconocida y brillante transformación urbana que ha sufrido la ciudad de Medellín en los últimos tiempos.

Tras Osorio, Clara Mejía y María Pía Fontana realizaron una cuidada presentación de las figuras de Rogelio Salmona y el equipo de arquitectos José María Obregón y Pablo de Valenzuela, estableciendo una comparación entre dos de sus obras más conocidas: el proyecto de las Torres del Parque de Salmona y el conjunto residencial Bavaria de Obregón y Valenzuela, estratégicamente situadas las dos en las inmediaciones del Parque de la Independencia de Bogotá.

José María Ezquiaga, Catedrático en la ETSAM y Decano del COAM, clausuró la Bienal con una conferencia titulada La arquitectura como espacio público: la experiencia de Colombia en la que reflexionó sobre el papel de la arquitectura en la historia reciente de Colombia como agente principal en la definición, creación y recuperación del espacio público para la ciudad. Un cambio radical unido a otro cambio radical en el clima de convivencia del país, con un horizonte de paz y de esperanza y que apuesta por el valor del espacio público como espacio de convivencia con una renovada atención a las clases populares con propuestas eficientes de movilidad, insertando grandes equipamientos educacionales o culturales en áreas marginales en un intento eficaz por reducir las tremendas desigualdades sociales.

Hasta aquí probablemente nada nuevo y desconocido, salvo la constatación, que el tiempo ha vuelto a demostrar, de que la arquitectura sigue siendo en manos de las administraciones una extraordinaria herramienta para el progreso social.

Estas fueron las conferencias temáticas que acompañaron a la BAL y que tuvieron lugar en el Palacio del Condestable de Pamplona, donde también estaba montada la exposición con los trabajos de los quince equipos de bienalistas seleccionados, la muestra de obra reciente de diecisiete bienalistas anteriores y la exposición monográfica sobre los últimos cien años de arquitectura en Colombia. La exposición, de carácter itinerante, pudo verse en junio y julio en las Arquerías de los Nuevos Ministerios de Madrid. 


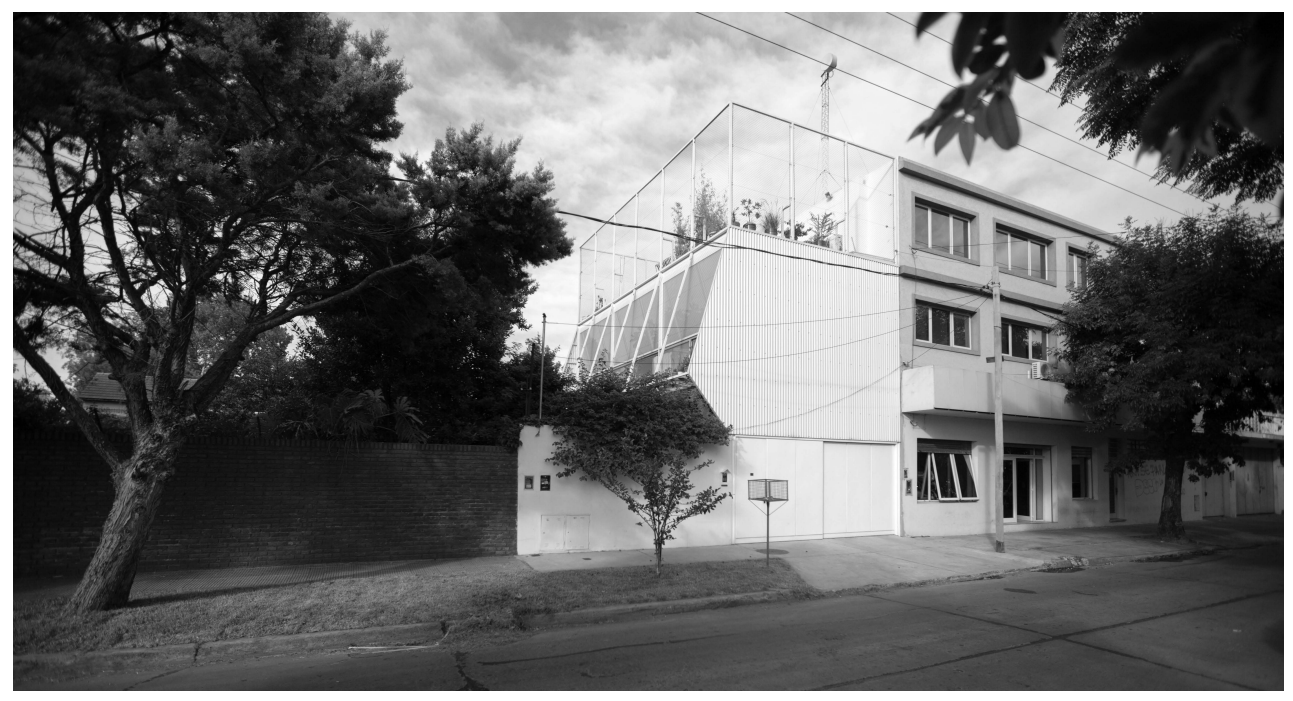

3

Paralelamente, en el Campus de la Universidad de Navarra, en su Escuela de Arquitectura tuvieron lugar durante las mañanas las conferencias en las que los jóvenes equipos de arquitectos latinoamericanos seleccionados mostraron su obra. Quince equipos de siete países (México, Brasil, Argentina, Chile, Colombia, Ecuador y Perú), seleccionados entre numerosos candidatos, presentaban, en muchos de los casos por primera vez en Europa, su obra construida, en la que la Bienal hace especial hincapié, y también sus propuestas para concursos y proyectos todavía no construidos. En esta edición por primera vez dos equipos, que ya habían participado en ediciones anteriores de la BAL: AToT y Adamo-Faiden vuelven a participar no ya como exbienalistas, sino como bienalistas de pleno derecho (cumplen la condición de las bases del certamen de no haber cumplido todavía los 40 años). Son dos equipos argentinos que se unen a otros tres conformando la nada sorprendente representación nacional más numerosa de la BAL.

Como corresponde, siendo Colombia el país invitado, fueron los equipos de este país los encargados de dar inicio a las conferencias: Taller EDU, de Medellín y Colectivo 720 de Cali. Dos equipos que coinciden públicamente por primera vez en Pamplona, y cuyas propuestas responden al patrón de lo tratado anteriormente con respecto a la arquitectura colombiana. Son arquitecturas bien planteadas, bien diseñadas, con un gran sesgo social, especialmente acusado en caso del equipo de Medellín y en los que los procesos participativos asumen el liderazgo en la estrategia para el éxito social de las propuestas arquitectónicas y urbanas. La gestión de los procesos se convierte en la clave del éxito final. Colectivo 720 con propuestas como la UVA orfelinato "UVA de la imaginación" en los tanques de almacenamiento de agua de Medellín logra exaltar los valores icónicos de una infraestructura puesta al servicio público del barrio que la circunda. En su UVA El Paraíso, Taller EDU convierte en norma los diseños participativos mediante la metodológica construcción colectiva de ideas e imaginarios donde el ciudadano es el protagonista, generándole con ello un sentido de pertenencia, apropiación y sostenibilidad.

El equipo brasileño RIZOMA, de Belo Horizonte, dio muestras de una madura sensibilidad típicamente brasileña especialmente visible en sus propuestas de galerías de arte y servicios concebidas para el complejo museístico y botánico Inhotim en Minas Gerais. Sus compatriotas paulistas de SIAA abordaron proyectos escolares y docentes de gran escala con una dimensión tropical.

Los cinco equipos argentinos mostraron un nada sorprendente buen hacer confirmando una vez más la realidad de una brillante generación de jóvenes arquitectos que reivindican el hacer mucho con pocos medios. Las obras de Adamo-Faiden, en su mayoría variaciones con repetición de viviendas desarrolladas dentro de la manzana típica de Buenos Aires hacen de la construcción su seña de identidad, ofreciendo un modo contemporáneo de

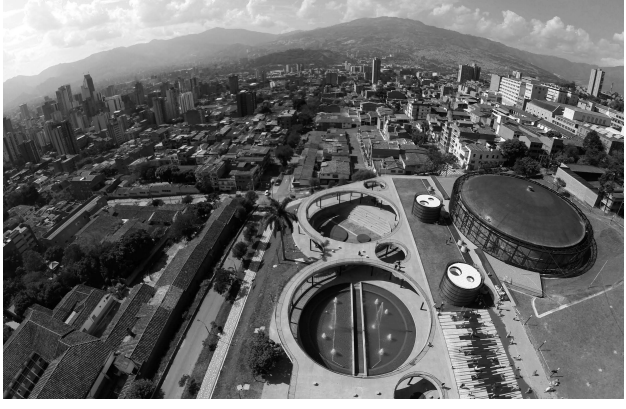

4

Fig. 3. Casa Martos, Adamo-Faiden, Buenos Aires, 2011 Fig. 4. UVA Orfelinato, Colectivo 720, Medellín, 2014 
Fig. 5. Cabaña Delta, AToT, Islas del Tigre, Argentina, 2014.

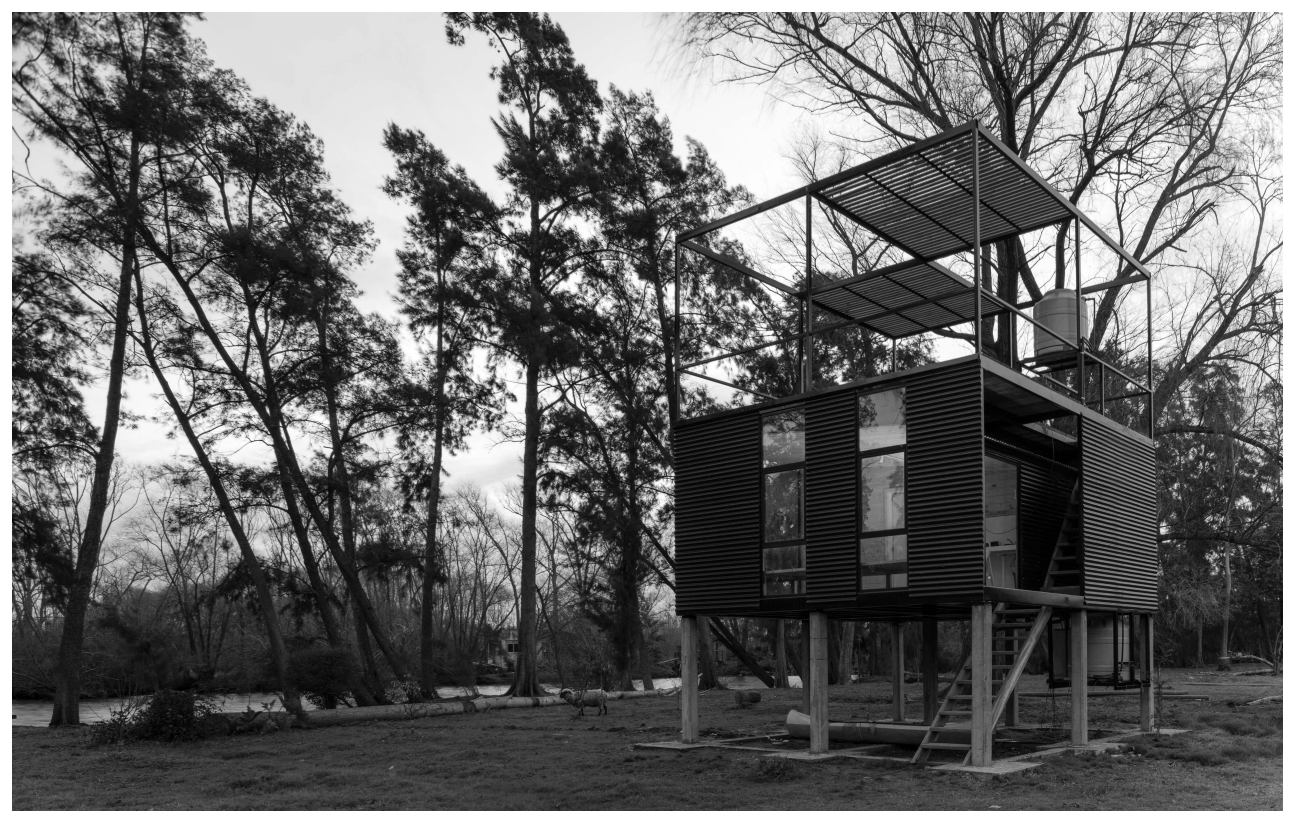

relacionarse con el entorno desde la respuesta a los nuevos hábitos en ecología doméstica unida a una actualización técnica de los sistemas de instalaciones. AToT, el equipo formado por Lucia Hollman y Agustín Moscato, también repitiendo presencia en la BAL, apuesta por la modulada liviandad, ofreciendo estructuras ligeras en paisajes exuberantes como en su proyecto de cabaña en el delta del Tigre. Sin movernos de Buenos Aires, Antonio Carrasco presentó una colección de viviendas en blanco y negro con evidentes referencias a la arquitectura moderna de los años 50 y planteadas como una reivindicación del oficio, el buen hacer artesano, el rigor constructivo, la claridad conceptual y el vigor poético. Torrado arquitectos, presento sus múltiples proyectos para el barrio de Puertos en la nueva ciudad de Escobar en la provincia de Buenos Aires. Un mismo sistema constructivo y espacial resuelve eficazmente los diferentes programas planteados en el desarrollo urbano del nuevo barrio. Desde Córdoba el equipo BLTARQ completa la representación argentina presentando varios proyectos de viviendas con el mismo sello de economía de medios y rigor constructivo, pero en el que se acentúa la investigación en los materiales y en los acabados y texturas superficiales.

Desde México vinieron Abraham Cota y CC Arquitectos. El primero formado en España nos mostró varias viviendas blancas, caracterizadas por la claridad formal y el rigor geométrico en las que la ineludible presencia del color nos recuerda que estamos en México. Manuel Cervantes Céspedes, o lo que es lo mismo CC Arquitectos nos sorprendió desde Ciudad de México con una abundante producción arquitectónica que ha sabido elaborar un lenguaje propio desde los riesgos de la sobreexposición de información a los que la arquitectura se haya expuesta; abordando diferentes escalas y registros en sus proyectos que dan como resultado una arquitectura rigurosa, técnicamente avanzada, reflejo de lo que la pujanza económica de un país puede hacer por la arquitectura cuando se aborda desde el rigor cultural y la tradición.

LLAMA, el estudio limeño formado por la arquitecta Mariana Leguía y el urbanista Angus Laurie ofreció un variado espectro de proyectos fruto del diverso origen de sus componentes (Perú y Canadá) y de la común formación en la London School of Economics. Proyectos que se combinan con la promoción y la construcción como medio de acceder a los encargos; y con una lógica racional en el proceso de generación de resultados que les lleva a ganar como arquitectos locales la ampliación del Museo de Arte de Lima (MALI) y a la construcción de una sofisticada casa puente en Ontario.

Desde Ecuador, Daniel Moreno Flores, nos ofreció una interesante muestra de la arquitectura que podríamos denominar étnica, es decir aquélla que como afirma José Manuel Pozo busca la versión más primitiva de la modernidad arquitectónica. En este caso una ar- 


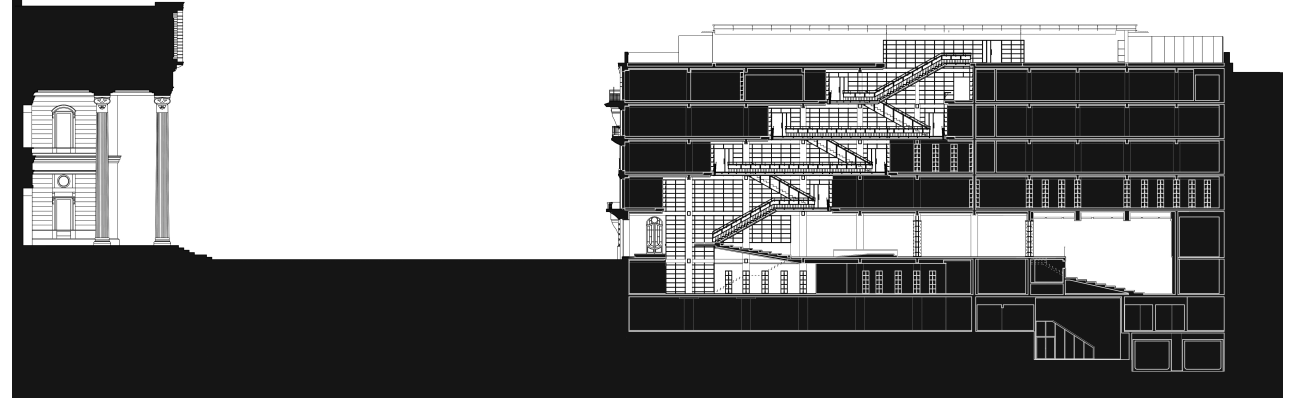

6

quitectura del reciclado que ofrece una ingeniosa reutilización de los materiales de deshecho como es el caso de la casa Contenedores o la casa El Carrizal reinterpretando las condiciones de la arquitectura local. Una arquitectura de pequeña escala, ambiciosa en sus planteamientos arquitectónicos y generosa en sus detalles, cercana en algún momento a la cultura del artefacto y con una fuerte carga pictórica y táctil en su paramentería.

Para acabar los arquitectos chilenos ofrecieron de nuevo una esperada muestra del buen hacer arquitectónico. El equipo Beals\&Lyon ofreció varios proyectos de gran escala, destacando la construida municipalidad de Nancagua con un interesante planteamiento urbano que apuesta por un espacio público de logrado carácter institucional. Sus otros proyectos como el de la nueva Biblioteca del Congreso Nacional o el edificio Metro en la Plaza de Armas traslucen una madurez conceptual y un oficio que confirman el conocido buen momento de la arquitectura chilena y van más allá de las maravillosas casas en paisajes espectaculares a las que estamos acostumbrados.

Alejandro SOFFIA, presentó una interesante colección de viviendas cuya característica principal es la del empleo de un material, el panel SIP, llevado hasta sus últimas consecuencias en cuanto a modulación y capacidad técnica; optimizando los costes de unas propuestas residenciales de ambiciosa condición constructiva y espacial. Con el declarado objetivo de lograr la mejor relación entre calidad de diseño y costo Soffia parte de la hipótesis de que la racionalización de la construcción debe definir las leyes del diseño. Sus planteamientos conceptuales y gráficos optan por una vía para la arquitectura similar a la de Ikea en el mobiliario; opción que en el campo de la arquitectura acota en corto sus propios límites.

Con los chilenos se concluye el repaso a lo que han sido los días de la bienal. Se han visto propuestas de gran interés y calidad, con programas que van desde la pequeña escala de las viviendas unifamiliares hasta una Central Intermodal como la de El Rosario en México obra de CC Arquitectos, con entornos que oscilan desde la periferia urbana, al centro histórico o al paisaje exuberante del delta del Tigre.

En la pasada Bienal de Venecia los comisarios del Pabellón de España apostaron por "arrojar una mirada optimista ante tantas arquitecturas inacabadas y sumidas en un estado de ruina fruto de la sinrazón de los últimos años, de la ausencia de reflexión y de la sustracción del factor tiempo de la fórmula para hacer arquitectura". En esta Bienal Latinoamericana hemos podido experimentar ese mismo optimismo ante una arquitectura con compromiso, con reflexión, con una actitud ética a la hora de la proporción entre los medios y los fines perseguidos, con un énfasis constructivo en los planteamientos. Una arquitectura con una opción preferencial por el más con menos que, en algún momento, nos podría recordar a las actitudes detrás de la brillante arquitectura de los 50 en España.

Opción preferencial por el más con menos que tiene mucho de sentido común y mucho de ambición y de intensidad en los contenidos y que desarrollada en un ambiente carente de la asfixiante normativa europea produce unos resultados que vistos desde esta periferia resultan envidiables.

Al igual que se hizo en la anterior edición, el programa de la V BAL se extendió a Barcelona y Madrid, donde varios de los equipos bienalistas pudieron presentar sus trabajos

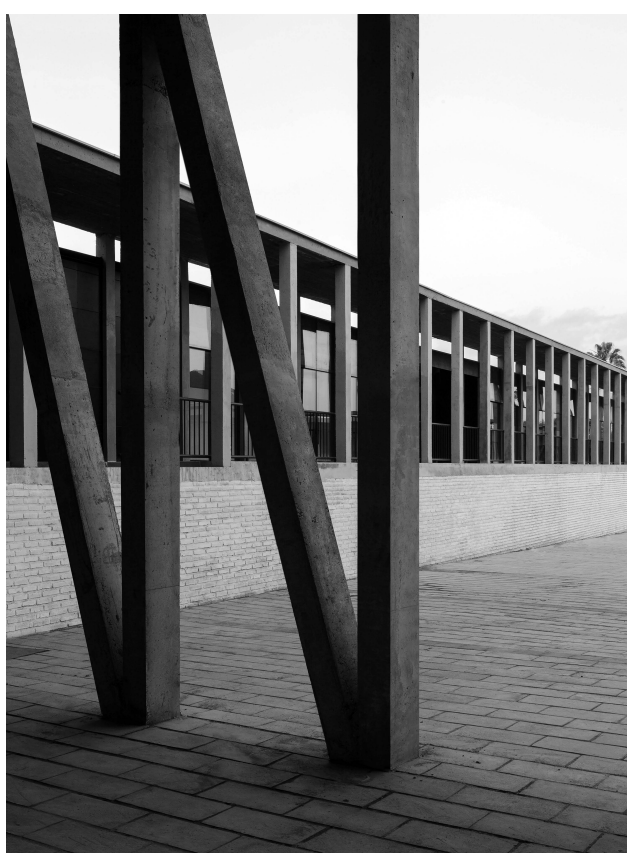

7

Fig. 6. Sección Biblioteca del Congreso, Beals\&Lyon y Matías Zegers, Chile, 2013.

Fig. 7. Municipalidad de Nancagua, Beals\&Lyon Arq, Chile, 2015. 
Fig. 8. Grupo AS20 y participantes BAL 2017 en la Escuela de Arquitectura de la Universidad de Navarra.

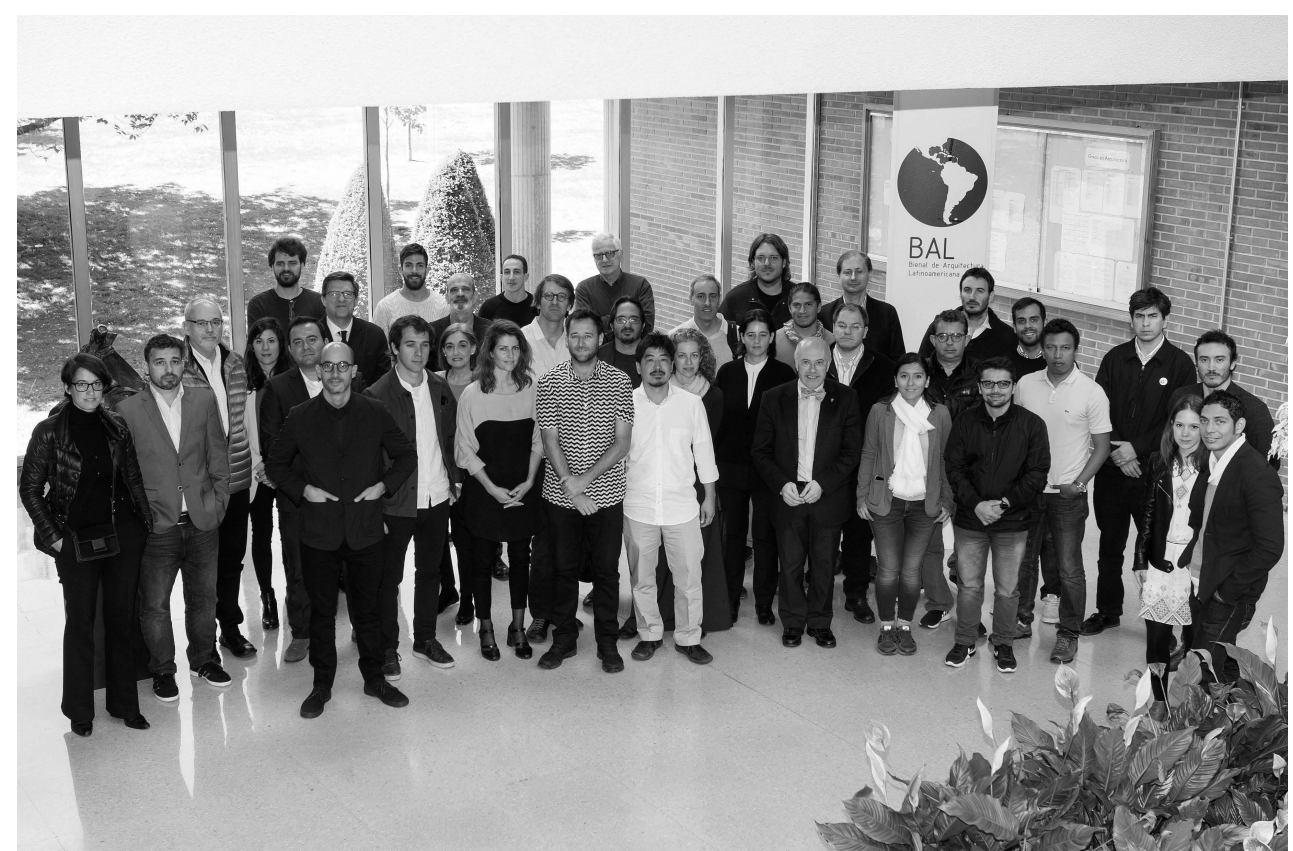

en la Escuela de Arquitectura y en el Colegio de Arquitectos de Barcelona y en Madrid, en la Escuela de Arquitectura. Un esfuerzo de difusión que incremente la validez del certamen y acentúa su función de escaparate del mejor presente de la arquitectura emergente latinoamericana. Cuenta además con un canal de difusión en Youtube en el que se pueden ver los videos de presentación de los equipos seleccionados y una selección de las conferencias. La Bienal de Arquitectura Latinoamericana es una iniciativa del grupo de investigación AS20 de la Escuela de Arquitectura de la Universidad de Navarra.

Rubén Labiano Novoa. Arquitecto por la Escuela de Arquitectura de la Universidad de Navarra (1993) y Doctor Arquitecto (2016). Dedicado al ejercicio libre de la profesión. Su obra ha sido merecedora de diversos premios y distinciones. Actualmente es Profesor asistente Doctor en la asignatura de Proyectos. Anteriormente ha sido Profesor Asociado en el Departamento de Proyectos, Urbanismo y Teoría e Historia de la Arquitectura de la Escuela de Arquitectura de la Universidad de Navarra. Visiting Scholar en Cornell University (USA) en el segundo semestre del curso 2013-14. Miembro del grupo de investigación AS20 sobre la arquitectura española de la segunda mitad del siglo XX. Sus líneas de investigación se centran en la arquitectura de ese periodo sobra la que ha escrito diversos artículos y participado en los tres últimos Congresos Internacionales de Historia de la Arquitectura Moderna Española organizados por la ETSAUN. Fruto de sus análisis es su tesis doctoral, Los sesenta años de arquitectura Fray Francisco Coello de Portugal, (1953-2013) defendida en enero de 2016. Ha sido secretario de la Bienal de Arquitectura Latinoamericana de Pamplona en sus tres últimas ediciones BAL 2013, BAL 2015 y BAL 2017. 